\title{
Investigating Grain Boundary Structures and Energetics of Rutile with Reactive Molecular Dynamics
}

${ }^{1}$ Patrick J. Shamberger*

2,3 Jennifer L. Wohlwend

${ }^{2}$ Ajit K. Roy

${ }^{4}$ Andrey A. Voevodin

${ }^{1}$ Department of Materials Science and Engineering; Dwight Look College of Engineering; Texas A\&M University; College Station, TX, 77843, USA

2 Nanoelectronic Materials Branch; Materials and Manufacturing Directorate; Air Force Research Laboratory; Wright Patterson AFB, OH 45459, USA

${ }^{3}$ Universal Technology Corporation; Dayton, OH 45459, USA

${ }^{3}$ Department of Materials Science and Engineering; University of North Texas; Denton, TX 76203, USA

Corresponding Author: Patrick J. Shamberger

e-mail: patrick.shamberger@tamu.edu

phone: (979) 458-1086 


\section{SUPPLEMENTARY INFO}

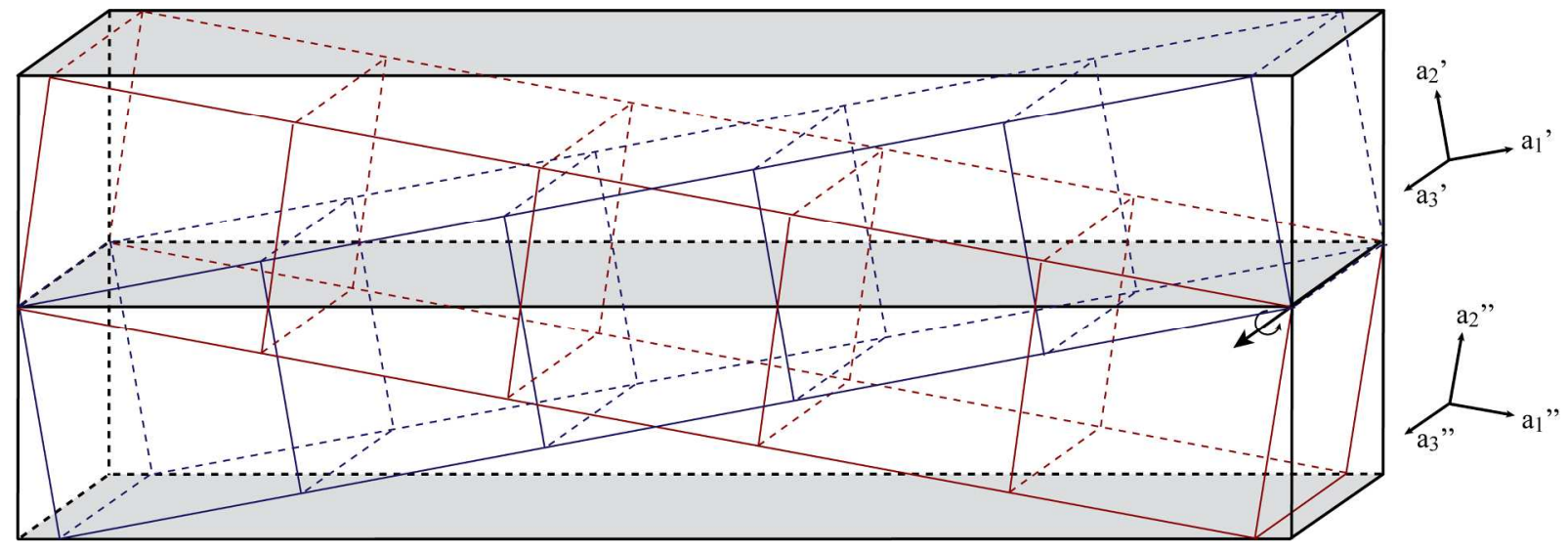

Figure S1. Computational cell used in this study, illustrating relationship between GB interfacial plane and computational cell volume necessary to ensure periodic boundary conditions. Thus, low-angle rotations around the rotation axis result in particularly large volume computational cells. 


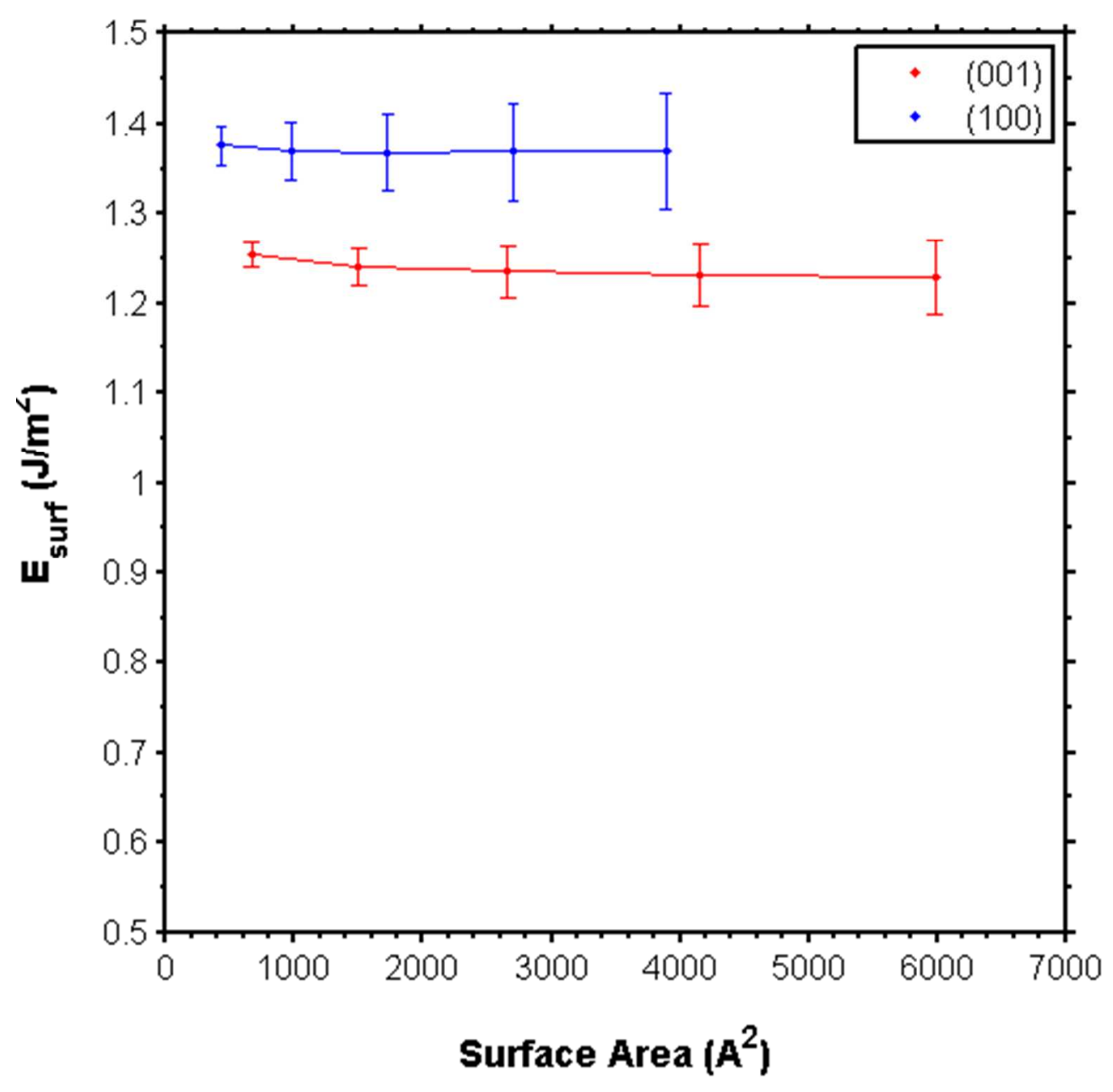

Figure S2. $\gamma^{\text {surf }}$ for low-index free surfaces $\left(\gamma_{(100)}, \gamma_{(001)}\right)$, with increasing $A_{\text {slab }}$. 

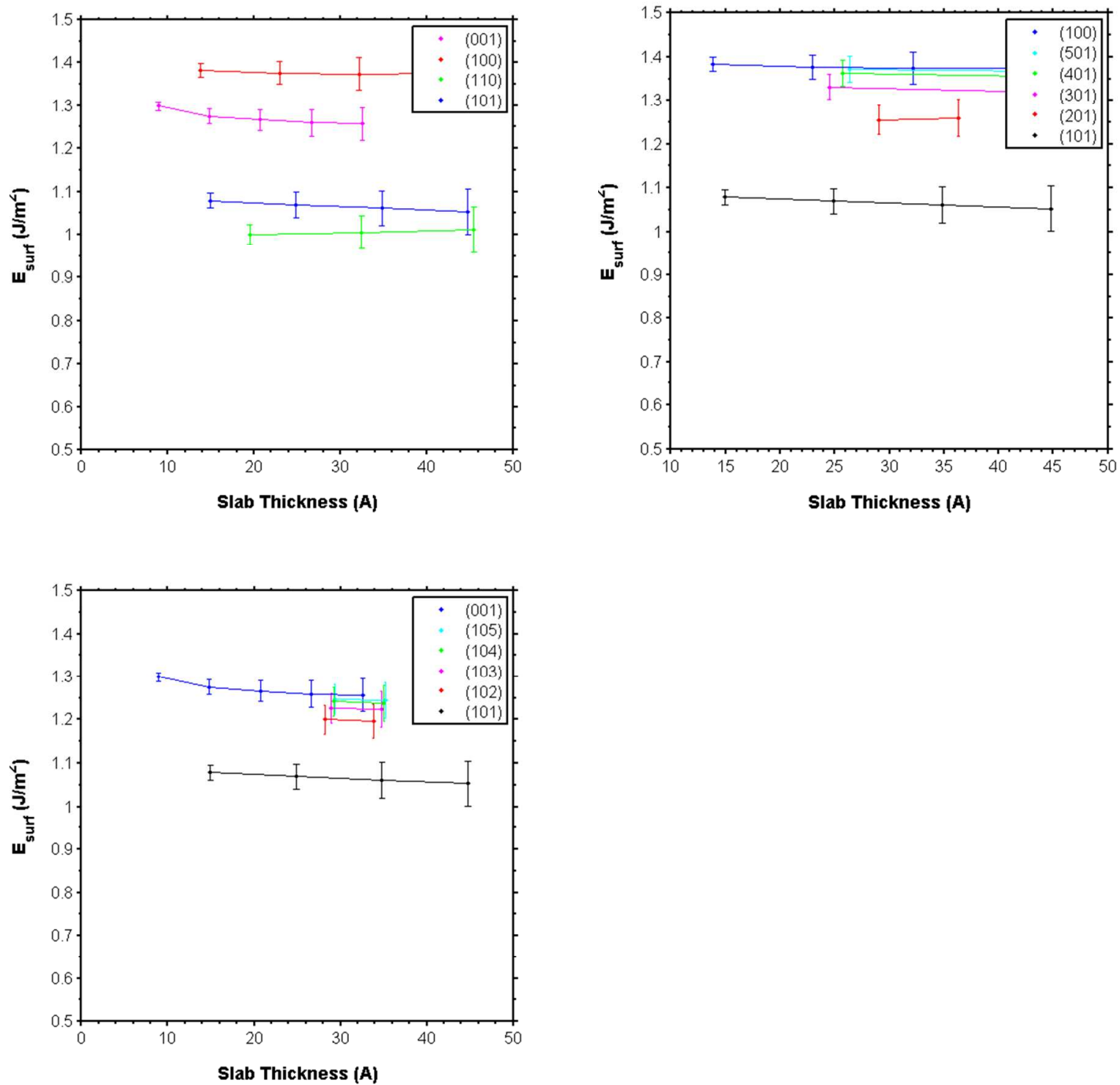

Figure S3. $\gamma^{\text {surf }}$ for a) low-index, b) $\{h 0 l\}$, and c) $\{h k 0\}$ free surfaces, with increasing $d_{\text {slab. In }}$ this study, $d_{\text {slab }}>2.5 \mathrm{~nm}$. 


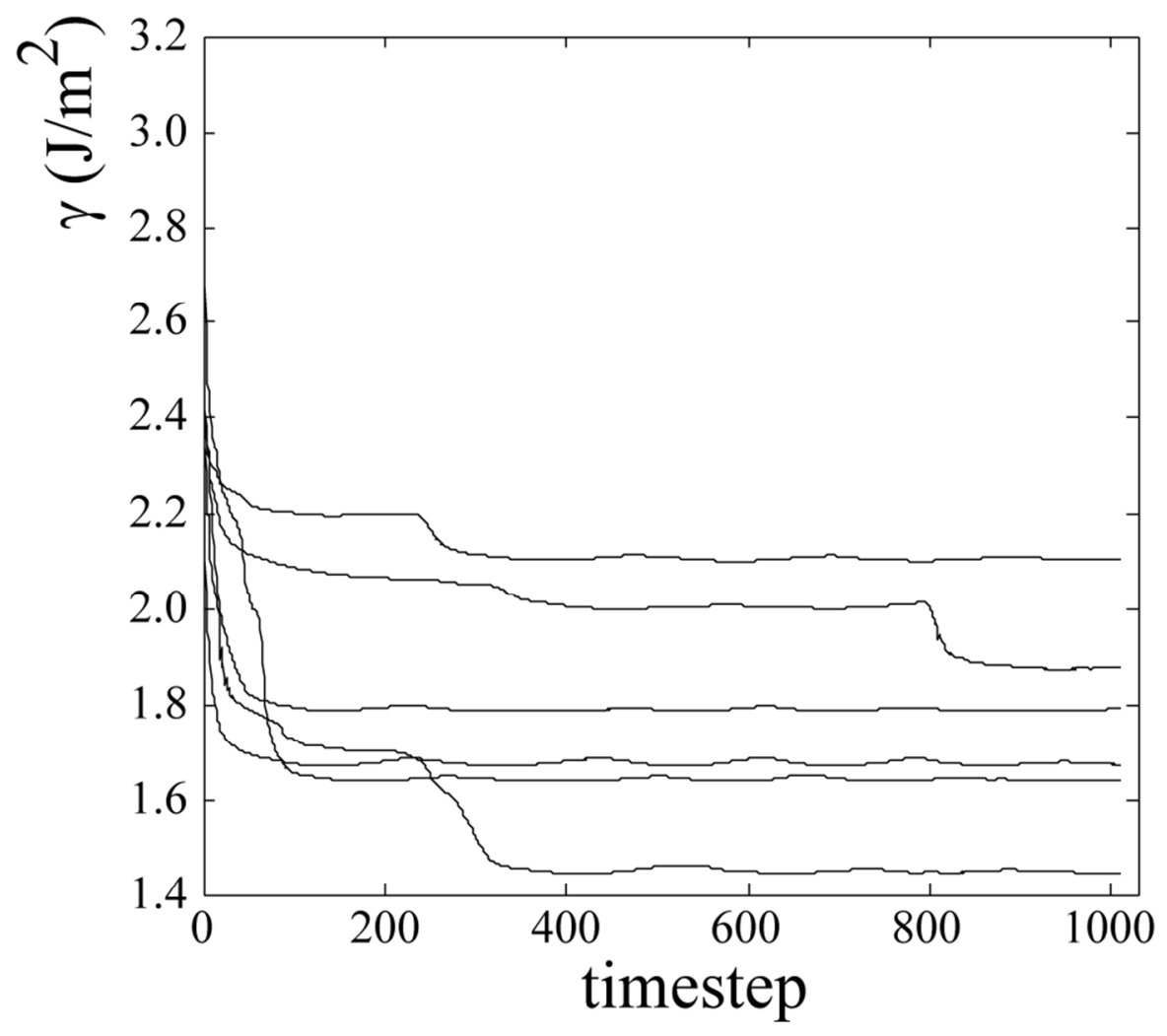

Figure S4. Example relaxation pathways for different initial displacements as indicated in Fig. 10b. in the text. Discrete relaxation steps are associated with individual atom or atoms moving to lower energy configurations on the GB. 